\section{Spontaneous alternation as a function of maze configuration*}

\author{
ROBERT J. DOUGLAS, DENIS MITCHELL, and DAVID KENTALA \\ University of Washington, Seattle, Wash. 98105
}

The tendency of the rat to alternate spontaneously was examined in mazes of different shapes. A typical $86.1 \%$ rate of alternation occurred in the T-maze, but the rate of $55.6 \%$ found in a maze with choice alleys side by side did not exceed chance. An even lower rate was found in a pilot study employing the latter maze. It was suggested that the crucial factor in spontaneous alternation is the spatial distinctiveness of the choice alleys and that alternation is not simply a matter of a choice between two alleys. The side-by-side maze configuration could be profitably employed as an alternative to the T-maze in learning studies in which spatial cues and/or alternation tendencies are confounding factors which bias either "learning speed" or group differences when one group differs from the other in either factor above.

A great advance was made in the analysis of rat behavior when Montgomery (1952) and Glanzer (1953) independently demonstrated that the phenomenon of spontaneous alternation was not based on a tendency to alternate body turns. That is, if rats were started from opposite ends of a + maze on each trial, they repeated body turns and thus alternated alleys. Unfortunately, however, the results were generally interpreted as having proven that rats do alternate visits to specific alleys. What is unfortunate is that the idea has persisted despite a great amount of disproof. It has been shown in carefully controlled experiments that rats simply do not alternate in response to visual, auditory, olfactory, tactile, proprioceptive, kinesthetic, or floor resonance cue differences, either in isolation or in combination (Douglas, 1966a). On the other hand, the rat will readily alternate spatial direction (not body turns) if given each of the two trials in a different $T$-maze, even with each maze in a different room. This two-maze alternation is abolished if the two mazes are aligned at right angles to each other, if the rat is made "dizzy" by turns in a horizontal plane, or if the vestibular system is malfunctioning due to middle-ear disease (Douglas, $1966 \mathrm{a}, \mathrm{b})$. The rat, in other words, behaves as if he defines an alley in terms of its spatial coordinates or direction. As far as alternation is concerned, the rat appears to ignore nonvestibular cues. The basic findings above have been confirmed and extended by others (e.g., Sherrick \& Dember, 1966a, b; Rosen \& Stein, 1969 ) and replicated dozens of times

*This research was supported in part by Grant MH-18760-01 to the senior author from NIMH, Department of Health, Education and Welfare.
The Ss were 12 adult hooded rats from the vivarium stock of the Psychology Department, University of
Washington. There were 7 males and 5 females, all individually housed with continuous access to food and water. Prior to testing, each was gentled by two handling sessions per day for 10 days.

APPARATUS
The Ss were tested in the three different maze configurations shown in Fig. 1. Each maze was bottomless, with the floor being the Formica surface of the table on which the maze was placed. Illumination was provided by a hooded 71/2-W light bulb suspended $14 \mathrm{in}$. above the choice point. A sliding door separated the startbox from the main alley, and sliding doors were located at choice alley entrances. At these entrances, there were inserts with holes $3 \frac{1}{2}$ in. in diam. In order to enter a choice alley, $S$ had to step through the hole. This procedure greatly facilitates scoring, as it makes alley entrance a discrete event, with Ss scored as having entered when their whole body was through the hole. Further, Ss tended to jump through the hole rather than linger near the entrance.

\section{PROCEDURE}

The procedure for a single test (two trials) was as follows: $\mathbf{S}$ was placed in the startbox of the maze scheduled for that session, and after 3 sec, the door to the main alley was raised. When $S$ had entered a choice alley, the door to that alley was lowered and $\mathrm{S}$ confined there for $30 \mathrm{sec}$. He was then removed and replaced in the startbox for an identical second trial. Each $\mathbf{S}$ was given one two-trial test per session. The sequence in which the three mazes were employed was determined randomly (drawing without

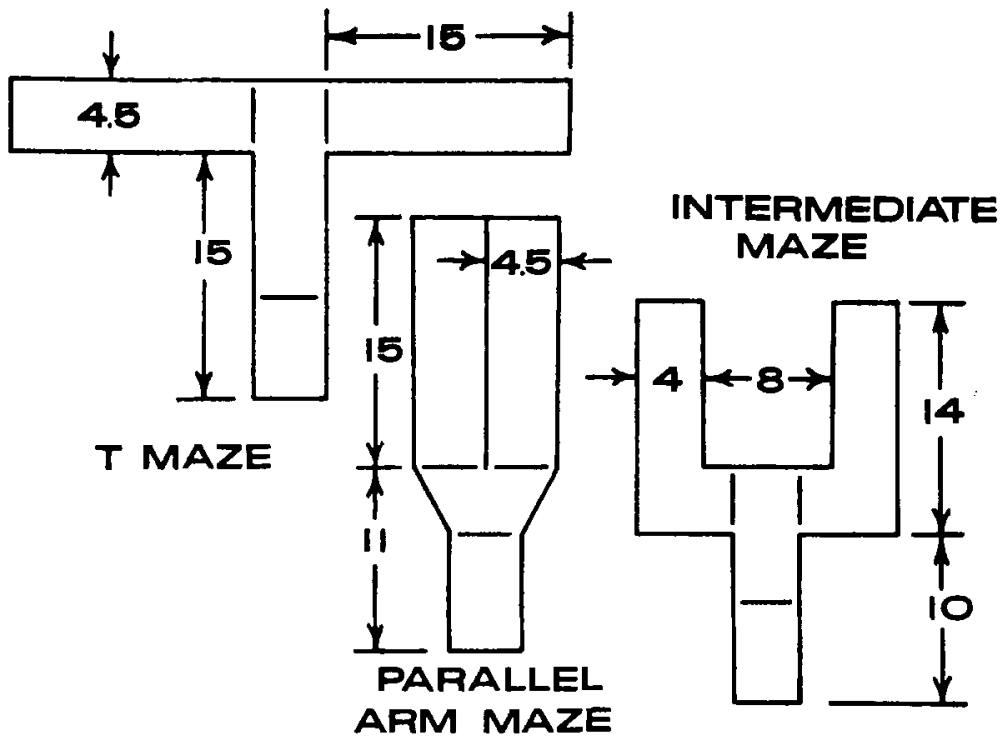

Fig. 1. The three maze configurations used in this study. Dimensions are given in inches. 
replacement), with a pair of Ss initially assigned to each of six possible three-maze sequences. The experiment was then replicated with each $S$ having a different sequence, and replications were continued until each $S$ had been tested with one of the six possible sequences. After completion, each rat had been tested six times in each of the three mazes, for a total of 18 tests per S. Since there were $12 \mathrm{Ss}$, a total of 72 tests were administered in each maze. Statistical evaluation of the results were carried out by giving each rat a score (0 to 6$)$ for each maze and using difference $t$ tests to compare mazes.

\section{RESULTS}

Behavior in one of the mazes differed radically from that in the other two. Alternation in the conventional T-maze occurred at an expected rate of $86.1 \%$, a figure comparable to an estimated population mean of $85 \%$, as based on literally thousands of tests with this stock. T-maze alternation was, of course, reliably above an estimated $50 \%$ chance rate $(t=10.5, p<.001)$. Results from the "intermediate" maze were essentially equivalent to the $\mathrm{T}$. The alternation rate was $80.6 \%$, a figure reliably above chance $(t=5.1$, $\mathrm{p}<.001$ ), but not, of course, reliably deviant from the $86.1 \% \mathrm{~T}$-maze rate. In marked contrast to these findings was the $55.6 \%$ alternation rate found in the "parallel arm" maze. Scores on this maze were not reliably above chance $(t=0.9$, n.s.), but they were reliably lower than scores found in the $\mathrm{T}$ - or intermediate mazes $(\mathrm{t}=4.2$, $\mathrm{p}<.01 ; \mathrm{t}=2.9, \mathrm{p}<.02$, respectively).

Essentially identical results were found with each replication, and in no case could an order effect be demonstrated. It seems clear, then, that the configuration of the maze makes a great deal of difference in determining whether alternation will or will not occur. When the two alleys are side by side (parallel-arm maze), the rat either does not alternate his choice of alleys or does so at a greatly reduced rate.

\section{DISCUSSION}

These results leave no room for doubt that spontaneous alternation is not merely due to the presence of two different alleys and an alternation of alley choice. The two alleys of the parallel-arm maze were as "distinct" intrinsically as were the two choice alleys in the other two mazes. The vast difference in alternation must then be due to something other than the presence of two different alleys, and we can conceive of no other explanation than that the two choice alleys must be spatially distinct if they are to elicit alternation. The results suggest that if alternation is based on vestibular cues, then two parallel side-by-side alleys are not sufficiently distinct, spatially, for the rat's vestibular system to distinguish them. The errors of the spatial coordinate system might be such that these two locations overlap in their "definition." It is not possible at this time to distinguish between two different interpretations. That is, we cannot at this point say whether the crucial factor is the angle between the two choice alleys or the distance between goals. This will be determined in the future by systematically varying the angle from the $0 \mathrm{deg}$ of the parallel-arm maze to the $180 \mathrm{deg}$ of the $T$ while holding alley length constant. Then angle will be held constant while length of alley varies. In any event, it is clear that the brain mechanisms and/or sensory systems involved in the powerful alternation tendency do not "work" in the parallel-arm configuration. This finding is based not only on the evidence reported here, but also on a previously completed pilot study in which only 19 of 40 rats alternated in the parallel-arm maze, with each $\mathrm{S}$ tested once.

It might be argued that our present results are due to a failure to "differentiate" the two alleys. In all mazes, both alleys were painted black. It remains possible that rats just might alternate in the parallel-arm maze if, say, one alley is painted black and the other white. This is doubtful because this "differentiation," when done in a T-maze, actually reduces the observed alternation rate (Douglas, 1966a). Further, even if this procedure should increase alternation in the parallel-arm maze, it would not "explain" the present data, because alternation occurred in the other two mazes at very high rates, despite the lack of visual differentiation.

Finally, the parallel-arm maze could prove to be very useful as a training apparatus. In the past, many investigators have, in fact, employed mazes of similar configuration (e.g., the Yerkes box), but not for reasons suggested by the present study. We suggest that the parallel-arm configuration should be employed in all cases where either spatial orientation or alternation are confounding factors. This includes nearly all procedures which normally employ the T-maze. It especially includes experiments in which the treatment given to one group changes the alternation tendency. For example, either large hippocampal lesions or the injection of scopolamine totally abolish spontaneous alternation (Douglas \& Isaacson, 1964,1966 ). It is, to say the least, difficult to compare T-maze learning performance between groups when they differ radically in their response probabilities prior to learning. A difference in "learning speed" is highly confounded in such cases by differences in experience in both alleys and by a powerful tendency in one group which is opposed to learning. These difficulties, and others as well, can be eliminated or reduced by use of the parallel-arm maze.

\section{REFERENCES}

DOUGLAS, R. J. Cues for spontaneous alternation. Journal of Comparative \& Physiological Psychology, 1966a, 62, 171-183.

DOUGLAS, R. J. Spontaneous aiternation and middle ear disease. Psychonomic Science, 1966b, 4, 243-244.

DOUGLAS, R. J., \& ISAACSON, R. L. Hippocampal lesions and activity Psychonomic Science, 1964, 1, 187-188.

DOUGLAS, R. J., \& ISAACSON, R. L. Spontaneous alternation and scopolamine. Psychonomic Science, $1966,4,283-284$.

GLANZER, $M$. The role of stimulus satiation in spontaneous alternation. Journal of Experimental Psychology, 1953,45, 387-393.

MONTGOMERY, $K$. c. A test of two explanations of spontaneous alternation. Journal of Comparative \& Physiological Psychology, 1952, 45, 287-293.

OLTON, D. S., \& ISAACSON, R. L Importance of spatial location in active avoidance tasks. Journal of Comparative \& Physiological Psychology, 1968, 65, 535-539.

ROSEN, J. J., \& STEIN, D. G. Spontaneous alternation beahvior in the rat. Journal of Comparative \& Physiological Psychology. $1969,68,420-426$.

SHERRICK, M. F., \& DEMBER, W. N. The tendency to alternate direction of movement as reflected in starting stem running speed. Psychonomic Science, $1966 a, 6,29-30$

SHERRICK, M. F., \& DEMBER, W. N Trial-two goal arm alternation to direction of movement in trial-one straight alley. Psychonomic Science, $1966 \mathrm{~b}, 6,317-318$. 